\title{
The Royal College of Ophthalmologists recommendations on screening for hydroxychloroquine and chloroquine users in the United Kingdom: executive summary
}

\author{
Imran H. Yusuf ${ }^{1} \cdot$ Barny Foot $^{2} \cdot$ James Galloway $^{3} \cdot$ Michael R. Ardern-Jones ${ }^{4} \cdot$ Sarah-Lucie Watson $^{5} \cdot$ Cathy Yelf $^{6}$. \\ Michael A. Burdon ${ }^{7}$ Paul N. Bishop ${ }^{8} \cdot$ Andrew J. Lotery $\mathbb{1}^{9}$
}

Received: 19 March 2018 / Accepted: 22 March 2018 / Published online: 11 June 2018

(c) The Royal College of Ophthalmologists 2018

\section{Introduction}

The Royal College of Ophthalmologists recently published recommendations for retinal screening in users of hydroxychloroquine and chloroquine in the United Kingdom [1]. This article forms a summary of the key components of the guideline. These recommendations involve a range of stakeholders including patients, Ophthalmologists and the hospital eye service, Rheumatologists, Dermatologists, General Practitioners, community optometry services, healthcare commissioners, and patient groups/ voluntary organisations who have had input into the development of the guideline.

The full guideline can be found online at: https://www. rcophth.ac.uk/standards-publications-research/clinicalguidelines/. The criteria used for grading evidence is specified in Table 1, and the recommendations and grade of evidence supporting them are detailed in Table $2 \mathrm{a}, 2 \mathrm{~b}, 2 \mathrm{c}$, 2d, 2e, 2f, 2g. (Fig. 1).Table 2c is a standardised screening protocol that applies to all patients.

Andrew J. Lotery

A.J.Lotery@soton.ac.uk

1 Oxford Eye Hospital, West Wing, John Radcliffe Hospital, Headington, Oxford OX3 9DU, UK

2 The Royal College of Ophthalmologists, 18 Stephenson Way, Kings Cross, London NW1 2HD, UK

3 Department of Rheumatology, King's College Hospital NHS Foundation Trust, Denmark Hill, London SE5 9RS, UK

4 Department of Dermatology, University Hospital Southampton NHS Foundation Trust, Tremona Road, Southampton, Hampshire SO16 6YD, UK
A comprehensive non-systematic review of hydroxychloroquine retinopathy which summarises the evidence at the time of guideline development is available [2].

\section{The need for an update to guidance}

Hydroxychloroquine is a drug increasingly used in the treatment of systemic lupus erythematosus, rheumatoid arthritis and other autoimmune disorders [2]. Emerging evidence from clinical studies indicates that hydroxychloroquine confers a survival benefit in patients with systemic lupus erythematosus [3], and is often used in combination with other disease modifying agents in rheumatoid arthritis [2]. The use of hydroxychloroquine is increasing with an estimated 11,000 new treatment initiations per year in England and Wales [4]. Hydroxychloroquine retinopathy was previously considered rare (prevalence of $0.5 \%$ on in patients taking the drug for 6 years [5]) probably because the diagnosis was made when patients became symptomatic with fundus abnormalities visible on clinical examination. Previous recommendations (RCOphth, 2009) did not support

5 Royal Berkshire Hospital, London Road, Reading, Berkshire RG1 $5 \mathrm{AN}, \mathrm{UK}$

6 The Macular Society, Crown Chambers, South St, Andover, Hampshire SP10 2BN, UK

7 Queen Elizabeth Hospital, Mindelsohn Way, Edgbaston, Birmingham B15 2GW, UK

8 Manchester Royal Eye Hospital, Manchester University Hospitals NHS Foundation Trust, Manchester, M13 9WL \& School of Biological Sciences, University of Manchester, Manchester M13 9PL, UK

9 Clinical and Experimental Sciences, Faculty of Medicine, University of Southampton, Southampton SO16 6YD, UK 
Table 1 Summary of recommendation grade

Grade Explanation

A At least one meta-analysis, systematic review, or RCT rated as $1++$, and directly applicable to the target population; or

A body of evidence consisting principally of studies rated as $1+$, directly applicable to the target population, and demonstrating overall consistency of results

A body of evidence including studies rated as $2++$, directly applicable to the target population, and demonstrating overall consistency of results; or Extrapolated evidence from studies rated as $1+$ + or $1+$

B A body of evidence including studies rated as $2+$, directly applicable to the target population and demonstrating overall consistency of results; or extrapolated evidence from studies rated as $2++$

C Evidence level 3 or 4; or extrapolated evidence from studies rated as $2+$

GPP Good practice points based upon consensual expert opinion where the evidence base does not support A-C grading

Table 2a Screening criteria: patient groups in whom screening is recommended

\begin{abstract}
Recommendation
All individuals who have taken hydroxychloroquine for greater than 5 years should receive annual screening for retinopathy.

All individuals who have taken chloroquine for greater than 1 year should receive annual screening for retinopathy.

All individuals taking hydroxychloroquine who have additional risk factors for retinal toxicity may be screened annually from the baseline visit or annual screening commenced before 5 years of treatment completed. This is to be decided by a Consultant ophthalmologist following the baseline visit.

Additional risk factors: Concomitant tamoxifen use, impaired renal function (estimated glomerular filtration rate of less than $50 \mathrm{ml} /$ $\min / 1.73 \mathrm{~m}^{2}$ ), dose of hydroxychloroquine greater than $5 \mathrm{mg} / \mathrm{kg} /$ day.

It is the responsibility of the prescribing physician (as per GMC guidelines) to refer patients eligible for screening to the local hospital eye service.

The referring clinician should be encouraged to complete a standardised referral proforma specifying the key clinical details relevant to screening for retinal toxicity. This will allow a determination of risk of toxicity and interpretation of test results.
\end{abstract}

Table 2b Screening protocol: baseline examination

Table 2c Screening protocol: screening tests

\begin{tabular}{lc}
\hline Recommendation & Grade \\
\hline $\begin{array}{l}\text { All patients planning to be on therapy long term ( } \geq 5 \text { years for hydroxychloroquine and }>1 \text { year for } \\
\text { chloroquine) should receive baseline examination ideally within } 6 \text { months of starting }\end{array}$ & C \\
hydroxychloroquine or chloroquine and definitely within 12 months. & \\
Baseline examination should include colour fundus photography and spectral domain optical & GPP \\
coherence tomography. & \\
$\begin{array}{l}\text { If the baseline examination demonstrates macular pathology, a baseline Humphrey 10-2 visual field } \\
\text { test may be undertaken. }\end{array}$ & GPP \\
\hline
\end{tabular}

Recommendation $\quad$ Grade

In addition to oral communication, written information about hydroxychloroquine retinopathy and screening for hydroxychloroquine retinopathy should be given to all patients.

All patients should undergo 10-2 Humphrey visual field testing (using a white stimulus), followed by pupillary dilation and imaging with both spectral domain optical coherence tomography (SDOCT) and widefield fundus autofluorescence (FAF).

Patients with abnormalities on widefield fundus autofluorescence with normal 10-2 visual field test results should undergo 30-2 visual field testing on another date.

Patients with persistent and significant visual field defects consistent with hydroxychloroquine retinopathy, but without evidence of structural defects on SD-OCT or FAF may be considered for multifocal electroretinography. 
Table 2d Interpretation of screening results
Recommendation $\quad$ Grade

No toxicity: No abnormalities suggestive of retinal toxicity detected on any test.

B

Possible toxicity: One test result (which in the case of visual fields should be reproducible) typical GPP of hydroxychloroquine retinopathy, but typical abnormalities not present in other tests.

Definite toxicity: Two test results (one subjective test and one objective test) with abnormalities

B
Table 2e Management of patients with possible retinopathy
Table 2f Management of patients with definite toxicity

Recommendation $\quad$ Grade

Patients with possible hydroxychloroquine retinopathy should continue drug treatment. This will reduce the risk of inappropriate treatment cessation.

Patients with one abnormal test result on retinal imaging (SD-OCT \& widefield FAF) but normal visual fields (including 30-2 protocol if appropriate) should return for annual review as per the screening schedule. This will reduce the risk of inappropriate treatment cessation.

Patients with persistent visual field abnormalities in the context of normal structural imaging (SDOCT \& widefield FAF) may be referred for multifocal electroretinography. Treatment should continue until the outcome of electrophysiology is known.

Recommendation Grade

A recommendation to stop hydroxychloroquine should be made to the prescribing physician to facilitate further discussion between specialist (for the treatment indication) and patient about the risks of stopping hydroxychloroquine and the options for alternative drug therapy.

Some description by the ophthalmologist of disease severity (mild, moderate, or severe) may be helpful to facilitate this discussion between patient and prescribing physician.

It would be inappropriate for ophthalmologists to stop hydroxychloroquine treatment.

Patients should be referred for appropriate support at the point of detection of hydroxychloroquine retinopathy. This may involve low vision or eye clinic liaison officer (ECLO) services, registration of visual impairment, and referral to local and/or national charities.

Patients who are drivers should be advised not to drive until an Esterman visual field test confirms it is legal to do so. The patient should inform the Driver Vehicle Licensing Agency (DVLA).

\section{GPP}

Table $2 \mathrm{~g}$ Organisation of services

\begin{tabular}{ll}
\hline Recommendation & Grade \\
\hline Screening for hydroxychloroquine retinopathy should take place in the hospital eye service. & GPP \\
Screening for hydroxychloroquine retinopathy may most effectively take place in virtual clinics \\
where visual field testing and dilated retinal imaging is undertaken before later being interpreted by \\
either by an ophthalmologist or an allied health professional under the supervision of a consultant \\
$\begin{array}{l}\text { ophthalmologist. } \\
\text { Written communication from the ophthalmologist indicating the outcome of a screening episode }\end{array}$
\end{tabular}

screening as evidence at the time suggested that the disease was very rare and there was no reliable test for detecting disease at a reversible stage [6]. However, modern retinal imaging techniques have been shown to be reliable in detecting pre-symptomatic retinopathy. Moreover, new data using modern retinal imaging techniques identify the prevalence of hydroxychloroquine retinopathy at around $7.5 \%$ in patients taking the drug for more than 5 years, increasing to 


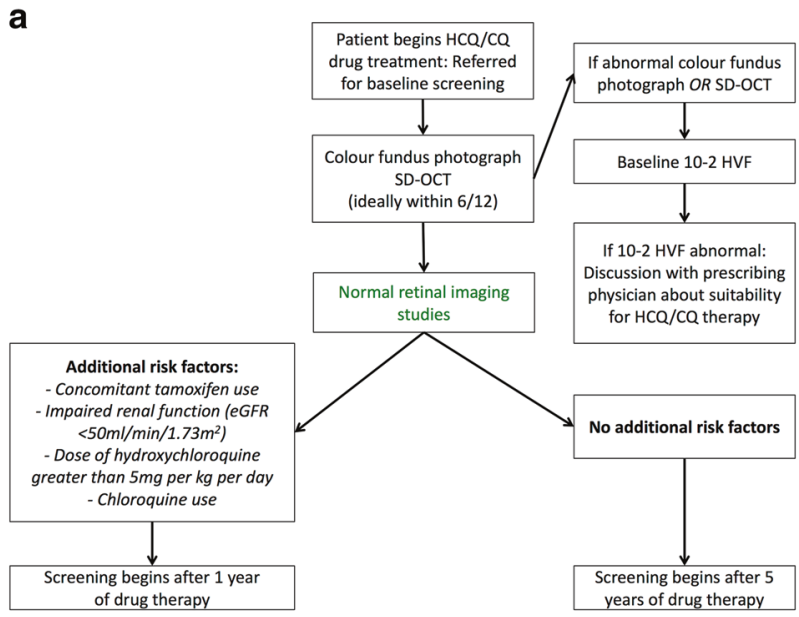

b

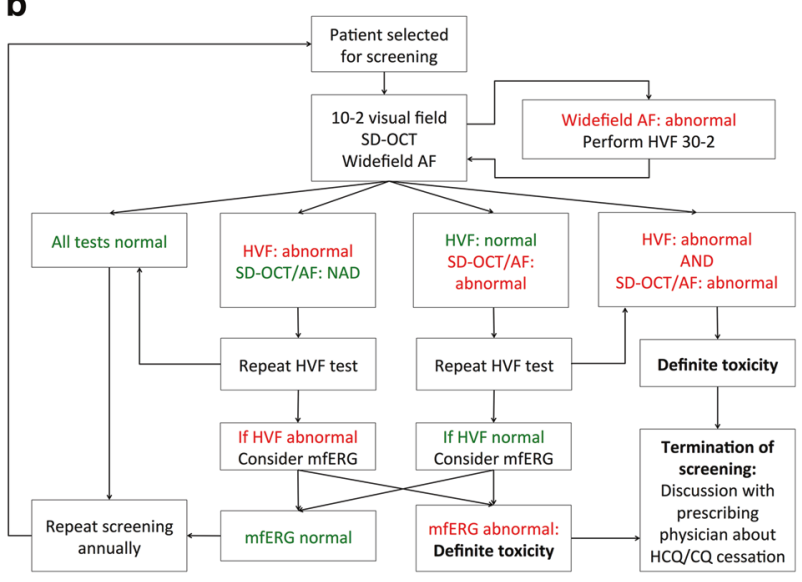

Fig. 1 A flow diagram summarising the recommended baseline tests (a) and the recommended screening algorithm (b)

20-50\% after 20 years [7]. These data suggest a large patient cohort with detectable pre-symptomatic hydroxychloroquine retinopathy in the United Kingdom who continue to take the medication.

It is recognised that most hospital eye services have modern retinal imaging technology (optical coherence tomography and fundus autofluorescence) and automated visual field testing which makes local screening possible. Such tests are generally acceptable to patients. Some ophthalmologists have recognised an increasing number of patients diagnosed with advanced hydroxychloroquine retinopathy who have developed symptoms [8]. It is also recognised that some opportunistic screening takes place in the hospital eye service led by clinicians in patients considered at risk of toxicity and in some motivated patients who seek referral for screening. Current screening protocols are likely to vary between centres with varying efficacy in detecting disease.

These factors in combination highlighted a need for an update to the guidance on screening for retinal toxicity in hydroxychloroquine and chloroquine users.

\section{Lay Summary}

Hydroxychloroquine is a safe and effective medication used to treat a variety of disorders. Long-term use of hydroxychloroquine can cause hydroxychloroquine retinopathy, a condition in which harmful effects on the retina can lead to permanent loss of vision affecting both eyes (retinopathy). Specialised retinal tests are able to detect early evidence of retinopathy before a patient notices visual changes. However, once hydroxychloroquine retinopathy results in noticeable loss of vision, the damage to the retina is permanent and often continues to get worse even if the medication is stopped. Hydroxychloroquine retinopathy becomes more likely the longer any individual is taking the medication and with higher doses of the medication. Looking for a particular condition in a person thought to be at risk, in order to detect it before symptoms develop to minimise the risk of harm is a process called "screening". The aim of screening for hydroxychloroquine retinopathy is to detect the earliest definite signs of the condition to allow those individuals to seek alternative medications in consultation with their prescribing doctor whilst allowing those unaffected to continue to benefit from the medication. Although most patients taking hydroxychloroquine will not develop retinopathy, around $7.5 \%$ of individuals taking hydroxychloroquine for more than 5 years may have signs of retinal damage detected on specialised tests.

\section{Methods}

The methods used in developing this guideline followed those specified in the RCOphth Clinical Guidelines Process manual and are outlined in full in the published guideline.

\section{Key research questions}

Research questions relevant to screening for hydroxychloroquine retinopathy were defined by the guideline development group:

\section{Epidemiology}

1. How many patients are currently receiving treatment and how many new patients are prescribed treatment each year?

2. What is the risk of toxicity retinopathy for patient subgroups (by disease and ethnic groupings)?

3. What is the risk of progression of toxicity retinopathy for patient sub-groups? 


\section{Screening}

1. Who should initiate the screening process?

2. Which patients require screening for hydroxychloroquine retinopathy?

3. When should patients be screened for hydroxychloroquine retinopathy?

4. What tests should be performed on patients as part of the screening schedule?

5. In what setting should screening for hydroxychloroquine retinopathy be performed?

6. What are the signs from screening tests that indicate hydroxychloroquine retinopathy?

7. What action should be taken for patients with hydroxychloroquine retinopathy?

8. What are the costs or economic implications of screening for hydroxychloroquine retinopathy?

9. What is the efficacy of screening protocols for detecting early hydroxychloroquine retinopathy?

10. Who is responsible for monitoring patient participation in screening?

11. What information should be obtained from referring clinicians to ophthalmologists at the initiation of screening?

12. What information should be communicated by ophthalmologists after a screening episode has been undertaken?

\section{Patient information}

1. What information should be given to patients at initial screening?

2. What information should be given to patients regarding treatment options at the point of detection of hydroxychloroquine retinopathy?

\section{Grading the level of evidence}

A pre-determined method for searching for evidence was followed, which involved Cochrane. The levels of evidence for each individual study were evaluated and graded by the guideline development group according to its strength using the Scottish Intercollegiate Guidelines Network framework (SIGN 50). The grade of each recommendation has been based upon the quality of the evidence and the potential for patient benefit (Table 1), rather than the clinical importance of the recommendation. This evidence was then evaluated in the context of the defined research questions to develop the recommendations and grade their strength. Using the evidence identified, the Guideline Development Group determined the guideline recommendations.
Key recommendations and Good Practice Points (GPP) for implementation

Tables $2 \mathrm{a}, 2 \mathrm{~b}, 2 \mathrm{c}, 2 \mathrm{~d}, 2 \mathrm{e}, 2 \mathrm{f}, 2 \mathrm{~g}$ specify the key components of the recommendations made, although additional recommendations relating to work commitments, patients unable to participate in the screening programme and termination of screening are specified in the full guideline.

\section{Safe dosing}

Current evidence suggests the highest risk of hydroxychloroquine retinopathy in patients taking a dose greater than $5 \mathrm{mg} / \mathrm{kg}$ per day [7]. Many patients take $400 \mathrm{mg}$ of hydroxychloroquine per day, which is a higher than recommended dose for any patient who weighs less than $80 \mathrm{~kg}$ [2]. All prescribers should be aware of the good practice recommendation that a dose of less than $5 \mathrm{mg} / \mathrm{kg}$ per day will reduce the risk of hydroxychloroquine retinopathy, although no absolutely safe dose has been identified [7]. Previous guidance suggested a safe dose defined on the basis of lean body weight [6]. However, there are a variety of methods of calculating lean body weight [2] and current evidence favours absolute body weight in defining risk of retinopathy [7]. Therefore, absolute body weight is both easier to measure and more informative. Safe dosing by general practitioners and hospital specialists is likely to reduce the incidence of hydroxychloroquine retinopathy in patients at risk [7]. A useful aide memoir for these guidelines for hydroxychloroquine is the $5 \times 5$ rule: ideally keep dosage $<5 \mathrm{mg} / \mathrm{kg} /$ day and screen after five years of drug use.

\section{Considerations for patients}

Education of patients at risk of hydroxychloroquine retinopathy is vital to ensure the risks of treatment are understood in the context of the significant benefits, the nature of screening tests, the screening schedule and how the outcome of screening is communicated and acted on. This will minimise any anxiety that may occur in patients at risk of retinopathy. In order to support the ongoing education of this patient group, the Macular Society has produced a patient information leaflet with input from the RCOphth guideline development group which is available online and should be distributed to all patients taking hydroxychloroquine and chloroquine [9]. Prescribing physicians should be aware of the screening recommendations so that appropriate referral to the hospital eye service can be made and that information given to patients about screening remains consistent.

Acknowledgements We thank our expert reviewers, Dr Ron Melles and Mr Alastair Denniston, college members who provided feedback 
on these draft guidelines and Iris Gordon from the Cochrane Eyes and Vision Group for assistance in searching the evidence basis.

Author contributions The Multidisciplinary guideline development group was chaired by AJL. IHY was involved in grading the evidence, writing the first draft of the guideline. BF represented The Royal College of Ophthalmologists and supported the guidance development process. $\mathrm{PNB}, \mathrm{MAB}$ and S-LW provided ophthalmic specialist clinical input. JG represented the British Society for Rheumatology and provided rheumatological specialist clinical input. MRA-J represented the British Association of Dermatologists and provided dermatological specialist clinical input. CY contributed as a Patient and Carer representation.

\section{Compliance with ethical standards}

Conflict of interest The authors declare that they have no conflict of interest.

\section{References}

1. Imran H. Yusuf, Barny Foot, James Galloway, Michael R. Ardern-Jones, Sarah-Lucie Watson, Cathy Yelf, Michael A. Burdon, Paul N. Bishop, Andrew J. Lotery. Royal College of Ophthalmologists Guideline Development Group - Hydroxychloroquine and Chloroquine Retinopathy: Recommendations on
Screening. https://www.rcophth.ac.uk/standards-publications-resea rch/clinical-guidelines/ Accessed 3 March 2018.

2. Yusuf IH, Sharma S, Luqmani R, Downes SM. Hydroxychloroquine retinopathy. Eye. 2017;31:828-45.

3. Alarcon GS, McGwin G, Bertoli AM, et al. Effect of hydroxychloroquine on the survival of patients with systemic lupus erythematosus: data from LUMINA, a multiethnic US cohort (LUMINA L). Ann Rheum Dis. 2007;66:1168-72.

4. Yates M, Malaiya R, Stack J, Galloway JB. Hydroxychloroquine use: the potential impact of new ocular screening guidelines. Eye. 2018;32:161-2.

5. Mavrikakis I, Sfikakis PP, Mavrikakis E, et al. The incidence of irreversible retinal toxicity in patients treated with hydroxychloroquine: a reappraisal. Ophthalmology. 2003;110:1321-6.

6. Richard Smith, Brian Bourke, Stephen Jones, Arul k Rajammal, Alan Silman. Hydroxychloroquine and Ocular Toxicity Recommendations on Screening. The Royal College of Ophthalmologists 2009.

7. Melles RB, Marmor MF. The risk of toxic retinopathy in patients on long-term hydroxychloroquine therapy. JAMA Ophthalmol. 2014;132:1453-60.

8. Latasiewicz M, Gourier H, Yusuf $\mathrm{IH}$, et al. Hydroxychloroquine retinopathy: an emerging problem. Eye. 2017;31:972-6.

9. Imran H. Yusuf, Cathy Yelf. Eye screening for patients taking hydroxychloroquine (Plaquenil ${ }^{\circledast}$ ): Macular Society. https://www. macularsociety.org/sites/default/files/resource/Hydroxychloroquine \%20-\%20access.pdf Accessed 1 March 2018. 